\title{
Kleene's three-valued logic and process algebra
}

\author{
Jan A. Bergstra ${ }^{\mathrm{a}, \mathrm{b}, 1}$, Alban Ponse ${ }^{\mathrm{a}, *}$ \\ ${ }^{a}$ University of Amsterdam, Programming Research Group, Kruislaan 403, 1098 SJ Amsterdam, The Netherlands \\ ${ }^{\mathrm{b}}$ Utrecht University, Department of Philosophy, Heidelberglaan 8, 3584 CS Utrecht, The Netherlands \\ Received 23 December 1997 \\ Communicated by H. Ganzinger
}

\begin{abstract}
We propose a combination of Kleene's three-valued logic and ACP process algebra via the guarded command construct. We present an operational semantics in SOS-style, and a completeness result. ( 1998 Elsevier Science B.V. All rights reserved.
\end{abstract}

Keywords: Process algebra; Three-valued logic; Guarded command; Design of algorithms; Concurrency; Formal languages

\section{Introduction}

In considering algorithms or programs in an operational manner, there is ample motivation to include a third truth value next to $T$ (true) and $F$ (false). For some illustrative references, see, e.g., [4,13]. Evaluation of the condition in a conditional construct, such as $\phi$ in

\section{if $\phi$ then $P$ else $P$,}

for some program $P$ may turn out divergent, or be distinguished as meaningless (e.g., a type clash, or division by zero). In such a case one certainly does not want to consider $P$ and if $\phi$ then $P$ else $P$ as equal. Typically, the principle of the excluded middletertium non datur-is not anymore acceptable. Of course, if $\phi$ then $P$ else $P$ and if $\neg \phi$ then $P$ else $P$ should be considered the same.

In this paper we view process expressions with conditions as a vehicle to describe concurrent algorithms, and consider the question how to deal with a third

\footnotetext{
* Corresponding author. Email: alban@wins.uva.nl.

${ }^{1}$ Email: janb@wins.uva.nl.
}

truth value $\mathrm{D}$, expressing divergence. This value is inspired by Kleene [15], in which it is called undefined, and is used to reason about partial recursive predicates being either undefined, true, or false. We rather use 'divergence' instead of 'undefined', as for example a type clash in a program is a kind of undefinedness that we want to distinguish from divergence. Naturally, $\neg \mathrm{D}=\mathrm{D}$, for divergence in the evaluation of a condition also implies divergence of its negation (cf. $\phi$ in if $\phi$ then $P$ else $P$ and if $\neg \phi$ then $P$ else $P$ ).

We shortly recall the combination of process algebra and logic via the guarded command, an operation which stems from [11], and was introduced in process algebra with two-valued logic in [2] with the following typical laws where $\phi: \rightarrow_{-}$is the guarded command resembling if $\phi$ then _:

$\mathrm{T}: \rightarrow x=x$,

$\mathrm{F}: \rightarrow x=\delta$,

$\phi: \rightarrow x+\psi: \rightarrow x=\phi \vee \psi: \rightarrow x$.

Here + denotes 'choice', and $\delta$ denotes 'inaction/ deadlock'. The constant $\delta$ is well known in ACP based 
approaches $[6,7,10]$, and is axiomatized by

$x+\delta=x \quad$ "inaction is not considered an alternative,

$\delta \cdot x=\delta \quad \ldots$ and is perpetual".

Here - represents "sequential composition". We involve the constant $\mathrm{D}$ with the axiom

$\mathrm{D}: \rightarrow x=\delta$

This preserves the three laws mentioned above in the present three-valued setting. Roughly, the idea is that if evaluation of a condition diverges, there is no point in considering it in the presence of an alternative, whereas it implies deadlock in case there are no alternatives. Now consider the derivations

$$
\begin{aligned}
x & =x+\delta \\
& =\mathrm{T}: \rightarrow x+\mathrm{D}: \rightarrow x \\
& =\mathrm{T} \vee \mathrm{D}: \rightarrow x, \\
\delta & =\delta+\delta \\
& =\mathrm{F}: \rightarrow x+\mathrm{D}: \rightarrow x \\
& =\mathrm{F} \vee \mathrm{D}: \rightarrow x .
\end{aligned}
$$

Clearly, the interpretation $\mathrm{D}: \rightarrow x=\delta$ leads to the logical consequence

$\mathrm{T} \vee \mathrm{D}=\mathrm{T}$,

and leaves only two options for the definition of $F \vee$ $D$, namely: $F \vee D \in\{F, D\}$. The only reasonable one seems $F \vee D=D .^{2}$ So we end up with $\neg, \vee$ and its dual $\wedge$ as defined by the following truth tables:
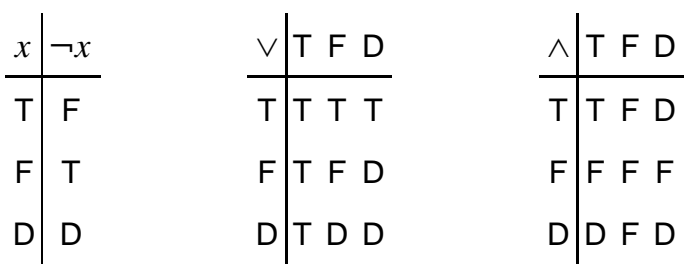

This precisely entails Kleene's three-valued logic as defined in [15], which we further call $\mathbb{K}_{3}$. (Notice that $\mathbb{K}_{3}$ is not functionally complete: one cannot define $f$ with $f(\mathrm{D})=\mathrm{F}$ and $f(v)=\mathrm{T}$ for $v \in\{\mathrm{T}, \mathrm{F}\}$.)

\footnotetext{
${ }^{2}$ By duality, the other option implies $T \wedge D=T$, which indeed seems a rather implausible interpretation of $\wedge$.
}

Structure of the paper. In the next section we shortly discuss $\mathbb{K}_{3}$. In Section 3 we combine this extension with ACP. In the next two sections we define an operational semantics and bisimulation equivalence, and we prove a completeness result.

\section{Kleene's three-valued logic with propositions}

Consider Kleene's three-valued logic $\mathbb{K}_{3}$ as introduced in the previous section (cf. [15,3]). An equational specification of $\mathbb{K}_{3}$ follows from [14], and is given in Table 1. As usual, $\wedge$ and $\vee$ are commutative and associative operations. In case we use proposition symbols from set $\mathbb{P}$, we shall write $\mathbb{K}_{3}(\mathbb{P})$, and for concise notation we shall identify $\mathbb{K}_{3}$ and $\mathbb{K}_{3}(\emptyset)$.

Let $\mathbb{T}_{3}^{D}=\{T, F, D\}$. In the following we describe a prototypical, generic occurrence of $\mathrm{D}$, starting from considerations that also apply to a two-valued setting. Consider the natural numbers

$\omega=\{0, S(0), S(S(0)), \ldots\}$,

and write $S^{0}(x)=x$ and $S^{k+1}(x)=S\left(S^{k}(x)\right)$. Let $f: \omega \rightarrow \mathbb{T}_{3}^{\mathrm{D}}$ be some arbitrary function. We define infinitary $f$-disjunction, notation $\bigvee f$, by

$\bigvee f=f(0) \vee \bigvee(f \circ S)$

The recursive definition of $\bigvee f$ implies computation of $f(0), f(S(0)), f\left(S^{2}(0)\right), \ldots$ until $f(n)=\mathrm{T}$ for some value $n$. In the particular case that for all $n \in \omega$, $f(n)=\mathrm{F}$, it makes sense to define $\bigvee f=\mathrm{D}$. We apply this idea in the following example.

Example 2.1. We define equality $\equiv: \omega \times \omega \rightarrow \mathbb{T}_{3}^{\mathrm{D}}$ as a binary infix function by

$0 \equiv 0=\mathrm{T}$,

$0 \equiv S(x)=\mathrm{F}$,

$S(x) \equiv 0=\mathrm{F}$,

$S(x) \equiv S(y)=x \equiv y$.

Next, we define the partial predecessor function pprd: $\omega \rightarrow \omega$ using auxiliary function $g: \omega \times \omega \rightarrow \omega$

$\operatorname{pprd}(x)=g(x, 0)$,

$g(x, y)= \begin{cases}y & \text { if } S(y) \equiv x, \\ g(x, S(y)) & \text { otherwise. }\end{cases}$ 
Table 1

Axiomatization of $\mathbb{K}_{3}$ with conjunction, disjunction, and implication.

\begin{tabular}{|c|c|c|c|}
\hline (K1) & $\neg \mathrm{T}=\mathrm{F}$ & (K6) & $x \wedge(y \wedge z)=(x \wedge y) \wedge z$ \\
\hline$(\mathrm{K} 2)$ & $\neg \mathrm{D}=\mathrm{D}$ & $(\mathrm{K} 7)$ & $\mathrm{T} \wedge x=x$ \\
\hline (K3) & $\neg \neg x=x$ & (K8) & $x \vee(x \wedge y)=x$ \\
\hline (K4) & $\neg(x \wedge y)=\neg x \vee \neg y$ & (K9) & $x \wedge y=y \wedge x$ \\
\hline (K5) & $x \rightarrow y=\neg x \vee y$ & $(\mathrm{~K} 10)$ & $x \wedge(y \vee z)=(x \wedge y) \vee(x \wedge z)$ \\
\hline
\end{tabular}

One easily sees that

$\operatorname{pprd}\left(S^{k+1}(x)\right) \equiv S^{k}(x)$.

Now consider the case of $\operatorname{pprd}(0)$. To model its computation, we define an auxiliary predicate $A u x$ as follows:

$\operatorname{Aux}(x, y, z) \Leftrightarrow g(x, y) \equiv z$.

The recursive definition of Aux follows easily from that of $g$, and falls within $\mathbb{K}_{3}(\mathbb{P})$ :

$$
\begin{aligned}
\operatorname{Aux}(x, y, z)= & (S(y) \equiv x \wedge y \equiv z) \\
& \vee \\
& (\neg(S(y) \equiv x) \wedge \operatorname{Aux}(x, S(y), z)) .
\end{aligned}
$$

In particular, $\operatorname{Aux}(0,0, z)$ models computation of $\operatorname{pprd}(0)$. We have

$$
\begin{aligned}
\operatorname{Aux}(0,0, z)= & (S(0) \equiv 0 \wedge 0 \equiv z) \\
& \vee \\
& (\neg(S(0) \equiv 0) \wedge \operatorname{Aux}(0, S(0), z)) .
\end{aligned}
$$

By $\mathrm{T} \wedge x=x$ and $S(x) \equiv 0=\mathrm{F}$, it follows that

$$
\begin{aligned}
\operatorname{Aux}(0,0, z)= & (S(0) \equiv 0 \wedge 0 \equiv z) \\
& \vee \\
& \left(S^{2}(0) \equiv 0 \wedge S(0) \equiv z\right) \\
& \vee \\
& \left(S^{3}(0) \equiv 0 \wedge S^{2}(0) \equiv z\right) \\
& \vee \\
& \cdots
\end{aligned}
$$

so, if $f=\lambda x .(S(x) \equiv 0 \wedge x \equiv z)$, we find

$\operatorname{Aux}(0,0, z)=\bigvee f$.

Furthermore, we have for each $n$ that $f(n)=\mathrm{F}$ by axiom $S(x) \equiv 0=\mathrm{F}$. Hence
$\operatorname{Aux}(0,0, z)=\mathrm{D}$

and thus $g(0,0) \equiv z=\mathrm{D}$. The assumption that

$\operatorname{pprd}(0)=g(0,0)$

can be computed to some value $z$ leads to value $D$ of the predicate modeling its computation, irrespective of $z$. This motivates the following definitions:

$\operatorname{pprd}(0)=\mathrm{D}$,

$\omega_{\mathrm{D}}=\omega \cup\{\mathrm{D}\}$,

so pprd: $\omega \rightarrow \omega_{\mathrm{D}}$. In order to integrate this example with process algebra, we extend the domains of all defined functions to $\omega_{D}$ by taking

$S(\mathrm{D})=\mathrm{D}$,

$\mathrm{D} \equiv x=x \equiv \mathrm{D}=\mathrm{D}$,

$\operatorname{pprd}(\mathrm{D})=\mathrm{D}$.

We continue with this example after having combined $\mathbb{K}_{3}(\mathbb{P})$ with process algebra.

\section{Process algebra with $\mathbb{K}_{3}(\mathbb{P})$}

In the left column of Table 2 we present a slight modification of $\operatorname{ACP}(A, \gamma)$, the Algebra of Communicating Processes $[6,7,10]$. Here $A$ is a set of atomic actions, and $\gamma$ a communication function that is commutative and associative. We take $\gamma$ total on $A \times A \rightarrow A_{\delta}$, where $A_{\delta}=A \cup\{\delta\}$, and the communication merge | commutative (CMC) (by which (CM6) and (CM9), the symmetric variants of (CM5) and (CM8) [10], become derivable). In the right column additional axioms on pre-abstraction $\left(t_{I}\right.$, i.e., renaming of all actions in $I$ to action $t$ ), and guarded command are listed, where $\phi$ is taken from $\mathbb{K}_{3}(\mathbb{P})$. These axioms are parameterized by action set $A_{t}=A \cup\{t\}$. We mostly suppress the . 
Table 2

The axiom system $\operatorname{ACP}_{\mathrm{D}}\left(A_{t}, \gamma, \mathbb{P}\right)$, where $a, b \in A_{t \delta}, H, I \subseteq A_{t}$.

(A1)

$$
x+(y+z)=(x+y)+z
$$

(A2)

$$
\begin{aligned}
& x+y=y+x \\
& x+x=x
\end{aligned}
$$

$$
(x+y) z=x z+y z
$$

$$
\begin{aligned}
& \mathrm{T}: \rightarrow x=x \\
& \mathrm{~F}: \rightarrow x=\delta \\
& \mathrm{D}: \rightarrow x=\delta
\end{aligned}
$$$$
(x y) z=x(y z)
$$$$
x+\delta=x
$$

$$
\delta x=\delta
$$

$$
a \mid b=\gamma(a, b) \quad \text { if } a, b \in A_{t}
$$

(GC1) $\quad \phi: \rightarrow x+\psi: \rightarrow x=\phi \vee \psi: \rightarrow x$

(CM1) $\quad x \| y=(x \Perp y+y \Perp x)+x \mid y$

(GC2) $\quad \phi: \rightarrow x+\phi: \rightarrow y=\phi: \rightarrow(x+y)$

(CM2) $\quad a \Perp x=a x$

(CM3)

$$
a x \Perp y=a(x \| y)
$$

(CM4) $\quad(x+y) \llbracket z=x \Perp z+y \Perp z$

$$
\begin{aligned}
(\phi: \rightarrow x) y & =\phi: \rightarrow x y \\
\phi: \rightarrow(\psi: \rightarrow x) & =\phi \wedge \psi: \rightarrow x \\
\phi: \rightarrow x \Perp y & =\phi: \rightarrow(x \llbracket y)
\end{aligned}
$$$$
\text { (CMC) } \quad x|y=y| x
$$$$
\text { (CM5) } \quad a x \mid b=(a \mid b) x
$$

$$
\begin{aligned}
\partial_{H}(\phi: \rightarrow x) & =\phi: \rightarrow \partial_{H}(x) \\
t_{I}(\phi: \rightarrow x) & =\phi: \rightarrow t_{I}(x)
\end{aligned}
$$$$
\text { (CM7) } \quad a x \mid b y=(a \mid b)(x \| y)
$$

(CM8)

$$
(x+y)|z=x| z+y \mid z
$$

$$
\begin{aligned}
\partial_{H}(a) & =a \quad \text { if } a \notin H \\
\partial_{H}(a) & =\delta \quad \text { if } a \in H \\
\partial_{H}(x+y) & =\partial_{H}(x)+\partial_{H}(y) \\
\partial_{H}(x y) & =\partial_{H}(x) \partial_{H}(y)
\end{aligned}
$$

$$
\begin{aligned}
t_{I}(a) & =a \text { if } a \notin I \\
t_{I}(a) & =t \quad \text { if } a \in I \\
t_{I}(x+y) & =t_{I}(x)+t_{I}(y) \\
t_{I}(x y) & =t_{I}(x) t_{I}(y)
\end{aligned}
$$

in process expressions, and brackets according to the following rules: - binds strongest, $: \rightarrow$ binds stronger than $\|, \mathbb{L}, \mid$, all of which in turn bind stronger than + . We use

$\operatorname{ACP}_{\mathrm{D}}\left(A_{t}, \gamma, \mathbb{P}\right)$

both to refer to this axiom system and the signature thus defined. We write

$\operatorname{ACP}_{\mathrm{D}}\left(A_{t}, \gamma, \mathbb{P}\right)+\mathbb{K}_{3}(\mathbb{P}) \vdash x=y$,

or shortly $\vdash x=y$, if $x=y$ follows from the axioms of $\operatorname{ACP}_{\mathrm{D}}\left(A_{t}, \gamma, \mathbb{P}\right)$ and $\mathbb{K}_{3}(\mathbb{P})$. The following derivabilities turn out to be useful:

\section{Lemma 3.1.}

(1) $\operatorname{ACP}_{\mathrm{D}}\left(A_{t}, \gamma, \mathbb{P}\right)+\mathbb{K}_{3}(\mathbb{P}) \vdash \phi: \rightarrow \delta=\delta$,

(2) $\operatorname{ACP}_{\mathrm{D}}\left(A_{t}, \gamma, \mathbb{P}\right)+\mathbb{K}_{3}(\mathbb{P}) \vdash \phi: \rightarrow x=\phi \vee \mathrm{D}: \rightarrow$ $x$.

Proof. As for (1), $\phi: \rightarrow \delta=\phi: \rightarrow \delta+\mathrm{T}: \rightarrow \delta=$ $\phi \vee \mathrm{T}: \rightarrow \delta=\mathrm{T}: \rightarrow \delta=\delta$.

As for (2), $\phi: \rightarrow x=\phi: \rightarrow x+\delta=\phi: \rightarrow x+\mathrm{D}: \rightarrow$ $x=\phi \vee \mathrm{D}: \rightarrow x$.

We end this section by using the functions defined in Example 2.1 in a process algebraic setting. 
Example 3.2. Recall the data type $\omega_{\mathrm{D}}$, and consider the following counter-like process with parameter in $\omega_{\mathrm{D}}$ :

$$
\begin{aligned}
C(x)= & r(\text { up }) \cdot C(S(x))+r(\text { down }) \cdot C(\operatorname{pprd}(x)) \\
& +r(\text { set_zero }) \cdot C(0) \\
& +x \equiv 0: \rightarrow r(\text { is_zero }) \cdot C(x) .
\end{aligned}
$$

Here, action $r(u p)$ models "receive command to increase", action $r$ (down) represents "receive command to decrease", action $r$ (set_zero) can be used to reset the counter to $C(0)$, and action $r\left(i s_{-} z e r o\right)$ indicates that the counter value equals 0 . We find:

$$
\begin{aligned}
& \begin{aligned}
C(\mathrm{D})= & r(\text { up }) \cdot C(\mathrm{D})+r(\text { down }) \cdot C(\mathrm{D}) \\
& +r(\text { set_zero }) \cdot C(0), \\
C(0)= & r(\text { up }) \cdot C(S(0))+r(\text { down }) \cdot C(\mathrm{D}) \\
& +r(\text { set_zero }) \cdot C(0)+r(\text { is_zero }) \cdot C(0), \\
C\left(S^{k+1}(0)\right)= & r(\text { up }) \cdot C\left(S^{k+2}(0)\right) \\
& +r(\text { down }) \cdot C\left(S^{k}(0)\right) \\
& +r(\text { set_zero }) \cdot C(0) .
\end{aligned}
\end{aligned}
$$

Clearly, this modeling is preferred to the case in which pprd is replaced by $\operatorname{prd}: \omega \rightarrow \omega$ with $\operatorname{prd}(0)=0$ and $\operatorname{prd}(S(x))=x$, which mixes up the number of $r($ down $)$ and $r(u p)$ actions in the case of $C(0)$.

\section{Operational semantics}

In this section we provide $\operatorname{ACP}_{\mathrm{D}}\left(A_{t}, \gamma, \mathbb{P}\right)$ with an operational semantics. Of course this semantics depends on interpretations of the propositions occurring in a process expression.

Assume a (non-empty) set $\mathbb{P}$ of proposition symbols, and let $w$ range over the valuations (interpretations) $\mathcal{W}$ of $\mathbb{P}$ in $\mathbb{T}_{3}^{\mathrm{D}}$. In the usual way we extend $w$ to $\mathbb{K}_{3}(\mathbb{P})$ :

$w(c) \triangleq c \quad$ for $c \in\{\mathrm{T}, \mathrm{F}, \mathrm{D}\}$,

$w(\neg \phi) \triangleq \neg(w(\phi))$,

$w(\phi \diamond \psi) \triangleq w(\phi) \diamond w(\psi) \quad$ for $\diamond \in\{\wedge, \vee\}$.

It follows that if

$\models w(\phi)=w(\psi)$

for all $w \in \mathcal{W}$, then $\models \phi=\psi$, and thus $\vdash \phi=\psi$.
In Table 3 we give axioms and rules that define transitions

${ }_{-} \stackrel{w, a}{\longrightarrow} \_\subseteq \operatorname{ACP}_{\mathrm{D}}\left(A_{t}, \gamma, \mathbb{P}\right) \times \operatorname{ACP}_{\mathrm{D}}\left(A_{t}, \gamma, \mathbb{P}\right)$

and unary "tick-predicates" or "termination transitions"

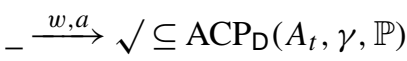

for all $w \in \mathcal{W}$ and $a \in A_{t}$. Transitions characterize under which interpretations a process expression defines the possibility to execute an atomic action, and what remains to be executed (if anything, otherwise $\sqrt{ }$ symbolizes successful termination). So, a process expression either resembles deadlock $(\delta)$, or defines outgoing transitions with labels taken from $\mathcal{W} \times A_{t}$.

The axioms and rules in Table 3 yield a structured operational semantics (SOS) based on the work described by Groote and Vaandrager in [12]. In particular, this SOS satisfies the so-called path-format (see Baeten and Verhoef [9]), going with the following notion of bisimulation equivalence:

Definition 4.1. Let $B \subseteq \operatorname{ACP}_{\mathrm{D}}\left(A_{t}, \gamma, \mathbb{P}\right) \times \operatorname{ACP}_{\mathrm{D}}\left(A_{t}\right.$, $\gamma, \mathbb{P})$. Then $B$ is a bisimulation if for all $P, Q$ with $P B Q$ the following conditions hold for all transitions ${ }_{-} \stackrel{l}{\longrightarrow}{ }_{-}$and ${ }_{-} \stackrel{l}{\longrightarrow} \sqrt{ }:$

- $\forall P^{\prime}\left(P \stackrel{-}{\longrightarrow} P^{\prime} \Longrightarrow \exists Q^{\prime}\left(Q \stackrel{l}{\longrightarrow} Q^{\prime} \wedge P^{\prime} B Q^{\prime}\right)\right)$, - $\forall Q^{\prime}\left(Q \stackrel{l}{\longrightarrow} Q^{\prime} \Longrightarrow \exists P^{\prime}\left(P \stackrel{l}{\longrightarrow} P^{\prime} \wedge P^{\prime} B Q^{\prime}\right)\right)$, - $P \stackrel{l}{\longrightarrow} \sqrt{ } \Longleftrightarrow Q \stackrel{l}{\longrightarrow} \sqrt{ }$,

Two processes $P, Q$ are bisimilar, notation

$P \leftrightarrow Q$,

if there exists a bisimulation $B$ containing the pair $(P, Q)$.

According to [9], bisimilarity is a congruence relation. It is not difficult to establish with induction on the size of terms that in the bisimulation model thus obtained all equations of Table 2 are true. Hence we conclude:

Lemma 4.2. The system $\operatorname{ACP}_{\mathrm{D}}\left(A_{t}, \gamma, \mathbb{P}\right)+\mathbb{K}_{3}(\mathbb{P})$ is sound with respect to bisimulation:

for all $P, Q \in \operatorname{ACP}_{\mathrm{D}}\left(A_{t}, \gamma, \mathbb{P}\right)$,

$\mathrm{ACP}_{\mathrm{D}}\left(A_{t}, \gamma, \mathbb{P}\right)+\mathbb{K}_{3}(\mathbb{P}) \vdash P=Q \quad \Longrightarrow \quad P \leftrightarrow Q$. 
Table 3

Transition rules in path-format.

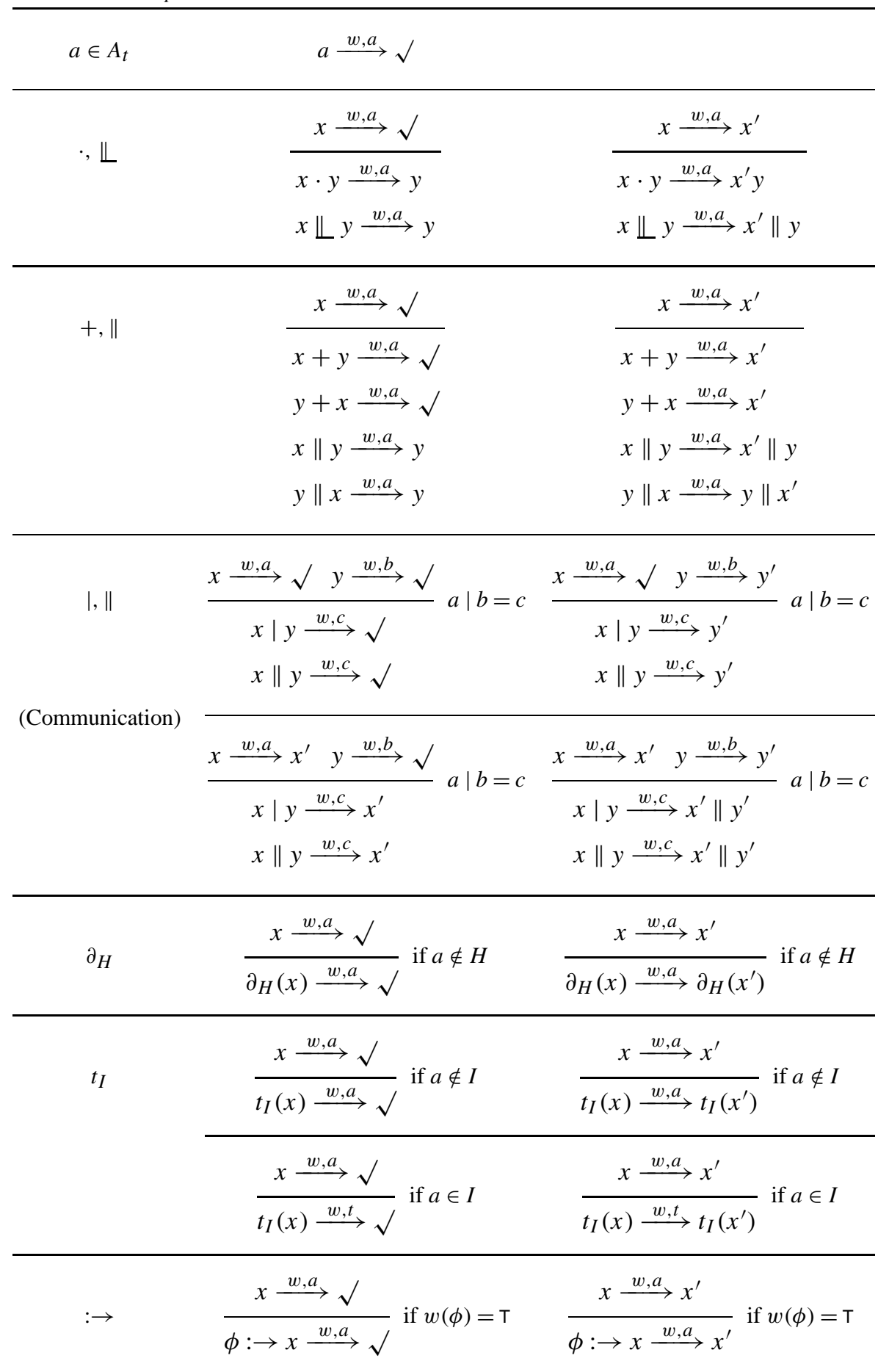




\section{Completeness}

In this section we prove completeness of $\operatorname{ACP}_{\mathrm{D}}\left(A_{t}\right.$, $\gamma, \mathbb{P})+\mathbb{K}_{3}(\mathbb{P})$, i.e.,

$P \leftrightarrow Q \Longleftrightarrow \operatorname{ACP}_{\mathrm{D}}\left(A_{t}, \gamma, \mathbb{P}\right)+\mathbb{K}_{3}(\mathbb{P}) \vdash P=Q$

Our proof is based on a representation of process expressions for which bisimilarity implies derivability in a straightforward way.

Definition 5.1. A process expression $P \in \operatorname{ACP}_{\mathrm{D}}\left(A_{t}\right.$, $\gamma, \mathbb{P})$ is a basic term if

$P \equiv \sum_{i \in I} \phi_{i}: \rightarrow Q_{i}$

where $\equiv$ is used for syntactic equivalence, $I$ is a finite, non-empty index set, $\phi_{i} \in \mathbb{K}_{3}(\mathbb{P})$, and $Q_{i} \in\{\delta, a, a R \mid$ $a \in A_{t}, R$ a basic term\}.

Lemma 5.2. All process expressions in $\operatorname{ACP}_{\mathrm{D}}\left(A_{t}, \gamma\right.$, $\mathbb{P})$ can be proved equal to a basic term.

Proof. Standard induction on term complexity.

For $a \in A_{t}$ and $\phi \in \mathbb{K}_{3}(\mathbb{P})$, the height of a basic term is defined by

$$
\begin{aligned}
& h(\delta)=0, \\
& h(a)=1, \\
& h(\phi: \rightarrow x)=h(x), \\
& h(x+y)=\max (h(x), h(y)), \\
& h(a \cdot x)=1+h(x) .
\end{aligned}
$$

Lemma 5.3. If $P$ is a basic term, there is a basic term $P^{\prime}$ with $\vdash P=P^{\prime}, h\left(P^{\prime}\right) \leqslant h(P)$, and $P^{\prime}$ has either the form

$\phi: \rightarrow \delta$,

or the form

$$
\sum_{i \in I} \psi_{i}: \rightarrow Q_{i}
$$

with

(i) for all $i, j \in I, Q_{i} \not \equiv \delta$, and $Q_{i}, Q_{j} \in A_{t} \Rightarrow$ $Q_{i} \not \equiv Q_{j}$ if $i \neq j$

(ii) for each $i \in I$ there is $w \in \mathcal{W}$ such that $w\left(\psi_{i}\right)=$ $\mathrm{T}$,

(iii) for no $i \in I$ and valuation $w, w\left(\psi_{i}\right)=\mathrm{F}$.
Proof. Assume

$P \equiv \sum_{i=1}^{n} \phi_{i}: \rightarrow Q_{i}$

for some $n \geqslant 1$. By Lemma 3.1(1) we may assume that $Q_{i} \not \equiv \delta$ for all $i \in\{1, \ldots, n\}$. With (GC1) we easily obtain that each single action occurs at most once. This proves property (i) of the form (2).

Next we consider all summands from $P$ for which no valuation makes the condition true. For each such summand $\phi_{i}: \rightarrow Q_{i}$ it holds that $\models \phi_{i}=\phi_{i} \wedge \mathrm{D}$, and thus $\vdash \phi_{i}=\phi_{i} \wedge \mathrm{D}$, by which

$$
\begin{aligned}
\vdash \phi_{i}: \rightarrow Q_{i} & =\phi_{i} \wedge \mathrm{D}: \rightarrow Q_{i} \\
& =\phi_{i}: \rightarrow\left(\mathrm{D}: \rightarrow Q_{i}\right) \\
& =\phi_{i}: \rightarrow \delta \\
& =\delta .
\end{aligned}
$$

In case all summands can be proved equal to $\phi_{j}: \rightarrow \delta$ in this way, we are done. In the other case we obtain

$\vdash P=\sum_{i=1}^{k} \phi_{i}: \rightarrow Q_{i}$

with $k \leqslant n$ (and possibly some rearrangement of indices), and for each $i \in\{1, \ldots, k\}$ there is a valuation $w$ with $w\left(\phi_{i}\right)=\mathrm{T}$. This proves property (ii), and preserves property (i) for $P$. Finally we define

$\psi_{i} \equiv \phi_{i} \vee \mathrm{D}$

$P^{\prime} \equiv \sum_{i=1}^{k} \psi_{i}: \rightarrow Q_{i}$.

By Lemma 3.1(2) we obtain

$\vdash P=P^{\prime}$

By definition of $\psi_{i}$ it follows that $w\left(\psi_{i}\right) \neq \mathrm{F}$ for all $w, i$, which proves property (iii) for $P^{\prime}$. (Properties (i) and (ii) are preserved for $P^{\prime}$.)

With these two lemma's we can prove completeness:

Theorem 5.4. The system $\mathrm{ACP}_{\mathrm{D}}\left(A_{t}, \gamma, \mathbb{P}\right)+\mathbb{K}_{3}(\mathbb{P})$ is complete with respect to bisimulation.

Proof. Let $P_{1} \leftrightarrow P_{2}$. By soundness, we may assume that both $P_{1}$ and $P_{2}$ satisfy the representation format 
defined in Lemma 5.3. We proceed by induction on $h=\max \left(h\left(P_{1}\right), h\left(P_{2}\right)\right)$.

Case $h=0$. By Lemma 3.1(1), $\vdash P_{n}=\delta$ for $n=$ 1,2 , so $\vdash P_{1}=P_{2}$.

Case $h>0$. Let $P_{n} \equiv \sum_{i \in I_{n}} \psi_{n, i}: \rightarrow Q_{n, i}$ for $n=$ 1,2, so the $P_{n}$ satisfy form (2) given in Lemma 5.3. Furthermore, we may assume that for all $i \in I_{n}$, $Q_{n, i} \nRightarrow Q_{n, j}$ for $j \in I_{n} \backslash\{i\}$. For the case $Q_{n, i} \equiv$ $a R_{n, i}$ and $Q_{n, j} \equiv a R_{n, j}$ this follows by induction: $R_{n, i} \leftrightarrows R_{n, j}$ implies $\vdash R_{n, i}=R_{n, j}$, so $\vdash a R_{n, i}=$ $a R_{n, j}$, and thus (GC1) can be applied.

Now each summand of $P_{1}$ can be proved equal to one in $P_{2}$, and by Lemma 5.3, each such summand yields a transition for a certain $w \in \mathcal{W}$.

- Assume that $P_{1} \stackrel{w, a}{\longrightarrow} \sqrt{ }$ for some $w, a$. Thus $w\left(\psi_{1, i}\right)=\mathrm{T}$ for some unique $i \in I_{1}$. By $P_{1} \leftrightarrow P_{2}$, there is a unique $j \in I_{2}$ for which $P_{2} \stackrel{w, a}{\longrightarrow} \sqrt{ }$ and $\models \psi_{1, i}=\psi_{2, j}$ (the latter derivability follows from Lemma 5.3 and the non-bisimilarity of different summands). Thus

$\vdash \psi_{1, i}: \rightarrow a=\psi_{2, j}: \rightarrow a$.

- Assume that $P_{1} \stackrel{w, a}{\longrightarrow} R_{1, i}$ for some $w, a$ and unique $i \in I_{1}$. Thus $w\left(\psi_{1, i}\right)=\mathrm{T}$. By $P_{1} \leftrightarrow P_{2}$, there must be some unique $j \in I_{2}$ for which $P_{2} \stackrel{w, a}{\longrightarrow} R_{2, j}$ and $R_{1, i} \leftrightarrow R_{2, j}$, and for which $=$ $\psi_{1, i}=\psi_{2, j}$ follows from Lemma 5.3. By induction we find $\vdash R_{1, i}=R_{2, j}$, and therefore $\vdash a R_{1, i}=$ $a R_{2, j}$ and hence

$\vdash \psi_{1, i}: \rightarrow a R_{1, i}=\psi_{2, j}: \rightarrow a R_{2, j}$.

By the derivabilities above and symmetry, $\vdash P_{1}=P_{2}$ quickly follows.

\section{Conclusion}

The extension of process algebra with guarded command to a setting with Kleene's three-valued logic seems a modest one, and can be characterized as giving up the principle of the excluded middle, and hence giving up the identity

$x=\phi: \rightarrow x+\neg \phi: \rightarrow x$,

but otherwise no surprising identities arise: $\mathrm{D}$ and $\mathrm{F}$ often play the same role in guarded commands. This matches with the intuition that a process like

$(\mathrm{D}: \rightarrow a) \| b c$ equals $b c \delta$. The deadlock, caused by a divergence, is postponed until all alternative behaviour has been executed.

We have argued that divergence arises from considerations about partial predicates (cf. [15]), and can be involved in process algebra by $\mathrm{D}: \rightarrow x=\delta$. Of course, in the case that the process of evaluation is prominent in the algorithm represented as a process expression, evaluation rather should be modeled as a process (which possibly diverges) than as a condition.

\section{References}

[1] K.R. Apt, Ten years of Hoare's logic, a survey, Part I, ACM Trans. Programming Languages Systems 3 (4) (1981) 431483.

[2] J.C.M. Baeten, J.A. Bergstra, Process algebra with signals and conditions, in: M. Broy (Ed.), Programming and Mathematical Method, Proceedings Summer School Marktoberdorf, 1990 NATO ASI Series F, Springer, Berlin, 1992, pp. 273-323.

[3] J.A. Bergstra, I. Bethke, P.H. Rodenburg, A propositional logic with 4 values: true, false, divergent and meaningless, J. Appl. Non-Classical Logics 5 (1995) 199-217.

[4] H. Barringer, J.H. Cheng, C.B. Jones, A logic covering undefinedness in program proofs, Acta Inform. 21 (1984) 251269.

[5] S.D. Brookes, C.A.R. Hoare, A.W. Roscoe, A theory of communicating sequential processes, J. ACM 31 (3) (1984) 560-599.

[6] J.A. Bergstra, J.W. Klop, The algebra of recursively defined processes and the algebra of regular processes, in: A. Ponse, C. Verhoef, S.F.M. van Vlijmen (Eds.), Algebra of Communicating Processes, Utrecht 1994, Workshops in Computing, Springer, Berlin, 1995, pp. 1-25. An extended abstract appeared in: J. Paredaens (Ed.), Proceedings 11th ICALP, Antwerp, Lecture Notes in Computer Science, Vol. 172, Springer, Berlin, 1984, pp. 82-95.

[7] J.A. Bergstra, J.W. Klop, Process algebra for synchronous communication, Inform. and Comput. 60 (1-3) (1984) 109137.

[8] J.A. Bergstra, M.P.A. Sellink, Sequential data algebra primitives, Technical Report P9602b, Programming Research Group, University of Amsterdam, 1996.

[9] J.C.M. Baeten, C. Verhoef, A congruence theorem for structured operational semantics with predicates, in: E. Best (Ed.), Proceedings CONCUR 93, Hildesheim, Germany, Lecture Notes in Computer Science, Vol. 715, Springer, Berlin, 1993, pp. 477-492.

[10] J.C.M. Baeten, W.P. Weijland, Process Algebra, Cambridge Tracts in Theoretical Computer Science 18, Cambridge University Press, 1990.

[11] E.W. Dijkstra, A Discipline of Programming, Prentice Hall International, Englewood Cliffs, NJ, 1976. 
[12] J.F. Groote, F.W. Vaandrager, Structured operational semantics and bisimulation as a congruence, Inform. and Comput. 100 (2) (1992) 202-260.

[13] C.B. Jones, C.A. Middelburg, A typed logic of partial functions reconstructed classically, Acta Inform. 31 (5) (1994) 399-430.
[14] J. Kalman, Lattices with involution, Trans. Amer. Math. Soc. 87 (1958) 485-491.

[15] S.C. Kleene, On a notation for ordinal numbers, J. Symbolic Logic 3 (1938) 150-155. 